\title{
Estudiantes con alta capacidad: explorando su distribución según tipo de establecimiento educativo
}

\author{
Gifted Students: Exploring their Distribution According to the Type of Educational Institution
}

\author{
Gracia Navarro-Saldañaa, *, Gabriela Flores-Oyarzo ${ }^{\mathrm{a}}$, María-Gracia González Navarro ${ }^{\mathrm{a}}$ \\ aUniversidad de Concepción, Chile
}

\begin{abstract}
Resumen
Antecedentes: estudiantes con alta capacidad o potencial de talento académico, necesitan un entorno enriquecedor para alcanzar un desempeño sobresaliente, y fenómenos como la segregación escolar impactan negativamente la transformación del potencial intelectual en talento académico. Objetivo: comparar la distribución de estudiantes con alta capacidad de quinto básico a cuarto medio de la provincia de Concepción, Chile, provenientes de familias de nivel socioeconómico bajo, medio y alto, según el tipo de establecimiento educativo en que estudian. Método: participaron 650 estudiantes entre 11 y 17 años de edad, quienes respondieron el test de matrices progresivas de Raven, el cual permitió identificar el potencial intelectual según el puntaje de corte. Resultados: hay diferencias estadísticamente significativas en el potencial intelectual entre estudiantes de establecimientos públicos, particulares subvencionados y particulares pagados, así como diferencias en la distribución del potencial intelectual según el nivel educativo de los estudiantes y el nivel socioeconómico de sus familias. Conclusiones: al explorar la distribución de estudiantes con alta capacidad según el tipo de establecimiento educativo, hay una mayor concentración de estudiantes con alta capacidad en establecimientos educativos con familias de nivel socioeconómico alto y una menor concentración de estos en establecimientos educativos con familias de nivel socioeconómico bajo.
\end{abstract}

Palabras clave: alta capacidad; potencial de talento académico; nivel socioeconómico familiar; segregación escolar; tipos de establecimiento educativo.

\section{Para citar este artículo:}

Navarro-Saldaña, G., Flores-Oyarzo, G. y González, M-G. (2022). Estudiantes con alta capacidad: explorando su distribución según tipo de establecimiento educativo. Liberabit, 28(1), e500. https:// doi.org/10.24265/liberabit.2022.v28n1.02

\begin{abstract}
Background: Gifted students or students with academic potential need an enriching environment to achieve an outstanding performance. However, phenomena such as school segregation negatively affect the transformation of intellectual potential into academic talent. Objective: To compare the distribution of gifted students from the fifth grade of elementary school to the fourth year of high school in the Province of Concepción, Chile, coming from families of low, medium and high socioeconomic status, determined by the type of educational institution in which they study. Method: Six hundred fifty (650) students aged between 11 and 17 participated in the study and answered the Raven's Progressive Matrices Test, which allowed the identification of the intellectual potential based on the cut-off score. Results: Statistically significant differences were found in the intellectual potential among students from public, subsidized private and fee-paying private educational institutions, as well as differences in the distribution of the intellectual potential relative to the students' education level and their families' socioeconomic status. Conclusions: When exploring the distribution of gifted students according to the type of educational institution, there was a higher concentration of those students in educational institutions with families of high socioeconomic status, and a lower concentration in educational institutions with families of low socioeconomic status.
\end{abstract}

Keywords: giftedness; academic potential; family socioeconomic status; school segregation; types of educational institution.

Este es un artículo Open Access publicado bajo la licencia Creative Commons Atribución 4.0 Internacional. (CC-BY 4.0)

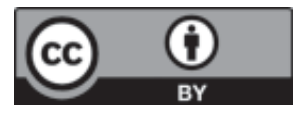

Universidad de San Martín de Porres, Lima - Perú http://ojs3.revistaliberabit.com 


\section{Introducción}

Para que las aptitudes de estudiantes con alta capacidad logren obtener un desempeño sobresaliente y desarrollen talento académico, requieren de condiciones individuales, factores sociales favorables $\mathrm{y}$ un entorno que responda efectivamente a sus necesidades particulares, actuando como un facilitador y protector que posibilita la expresión y desarrollo del potencial en latencia (Covarrubias, 2018; Freeman, 2000; Velasco \& Quiroga-Garza, 2018; Yun, 2020).

Los entornos educativos son importantes en el logro del desempeño académico de sus estudiantes, aunque, a la par, en ellos es probable que ocurran fenómenos que pueden obstaculizar oportunidades para el desarrollo del potencial intelectual, el bienestar y el aprendizaje de quienes se encuentran en entornos más desfavorecidos o vulnerables. Entre estos fenómenos se encuentra la segregación escolar.

Se denomina segregación escolar al fenómeno que ocurre cuando se observa una distribución desigual de estudiantes en los establecimientos educativos, conforme a sus cualidades personales, sociales y económicas (Duk \& Murillo, 2019; Morales, 2014; Murillo \& Martínez-Garrido, 2017; Murillo et al., 2018).

Por tanto, los catalizadores ambientales o contextuales juegan un rol fundamental en la transformación del potencial intelectual en desempeño sobresaliente, en el aprendizaje y en el bienestar de los y las estudiantes, encontrándose entre los entornos fundamentales la familia, la sociedad y el contexto educativo (Gagné, 2015; Renzulli, 2012). Dado el nivel de segregación social y escolar que caracteriza al contexto latinoamericano (Carrasco et al., 2014; Morales, 2014; Murillo \& Martínez-Garrido, 2017) y el efecto que tiene este fenómeno en la vida de niños, niñas y jóvenes en desarrollo (Duk \& Murillo, 2019; Méndez \& Gayo, 2019; Murillo et al., 2018), parece relevante explorar algunas diferencias entre estudiantes de nivel socioeconómico alto, medio y bajo asociado al tipo de establecimiento educativo en que cursan estudios.

\section{Alta capacidad: breve revisión del concepto}

El potencial humano puede entenderse como cualquier cualidad latente que pueda desarrollarse a través de experiencias (Yun, 2020). Al hablar de alta capacidad (AC), algunos autores refieren a una potencialidad intelectual superior latente en las personas y alude a un rendimiento intelectual superior que destaca por sobre la media, la cual, en tanto potencial, no implica necesariamente la expresión de talento cognitivo, intelectual ni académico (Covarrubias, 2018; Miguel \& Moya, 2011).

La AC guarda relación con el concepto de dotación propuesto por Gagné (2015), el cual refiere a una serie de dones o aptitudes que se encuentran de manera natural en las personas y que se expresan sin entrenamiento alguno. El talento, por su parte, refiere al dominio de estos dones, situando a la persona dentro del 10\% superior de su grupo etario (Gagné, 2015).

Arancibia (2009) plantea que un tipo específico de talento es el académico, el cual implica una habilidad o capacidad superior en aquello que la sociedad occidental considera como académico, por ejemplo, las matemáticas, las ciencias naturales, las ciencias sociales y las humanidades, «los talentos artísticos, los deportivos e interpersonales no quedan incluidos en dicha denominación», y que «los estudiantes con talento académico tienen características que los distinguen y de las cuales surgen sus necesidades educativas especiales» (p. 4).

Transformar la dotación en talento requiere de catalizadores interpersonales y ambientales (Gagné, 2015; González \& Domingues, 2015). Al estudiar la AC, los modelos sistémicos dan gran relevancia a la influencia contextual, social y ambiental (Pérez et al., 2017). Abraham Tannenbaum, en la década de los ochenta, propone un modelo psicosocial para comprender la dotación intelectual y el talento; planteando que para que el talento pueda emerger, no solo es relevante la capacidad o atributos personales, sino también los encuentros favorables con el ambiente, las experiencias enriquecedoras y 
las oportunidades que faciliten este proceso (Cornejo \& Benavides, 2013; Tourón, 2004). En la década de los noventa, Franz Mönks propone el modelo de interdependencia triádica, destacando como factores fundamentales para el desarrollo de las potencialidades intelectuales el entorno social inmediato como los pares, la escuela y la familia, y el macroambiente, destacando la situación sociopolítica, económica y cultural en que se encuentra el estudiante (Cornejo \& Benavides, 2013; Covarrubias, 2018; Gómez-Arizaga et al., 2019; Tourón, 2004).

Renzulli (2012), en su modelo de los tres anillos, sostiene que las altas capacidades se sitúan en la interacción de tres elementos: (1) capacidad intelectual sobre la media, (2) altos niveles de creatividad y (3) alto compromiso con la tarea.

El modelo propone que la persona con altas capacidades sería capaz de articular adecuada y equilibradamente aspectos intelectuales, como la inteligencia y la creatividad, y aspectos no intelectuales, como la voluntad, la motivación, el compromiso y la implicación en la tarea (Renzulli, 2012).

En esta misma línea, y abordando los planteamientos de los modelos sistémicos y contextuales, en Covarrubias (2018) se plantea que el talento solo puede desarrollarse gracias a la interacción favorable entre las condiciones individuales y los factores sociales, donde los contextos determinarían tanto las necesidades y los resultados del comportamiento humano, como los productos a ser considerados sobresalientes.

Para fines de este estudio, se entiende la alta capacidad (AC) como la presencia de capacidades o aptitudes intelectuales que se expresan espontáneamente y sin entrenamiento previo, constituyéndose como un potencial intelectual superior al $90 \%$ de la población del mismo rango etario. En tanto, el potencial requiere que la persona se comprometa con el aprendizaje, el entrenamiento y la práctica sistemática para transformar dicho potencial en desempeño sobresaliente o talento académico. Esta AC dota al estudiante de potencial para tener un desempeño sobresaliente en actividades académicas, el cual se expresará siempre y cuando sus necesidades educativas y socioafectivas sean atendidas, y esté en interacción con catalizadores ambientales que movilicen su motivación, esfuerzo y perseverancia (Navarro et al., 2021). Así, la AC se entiende como sinónimo de potencial de talento académico y se diferencia del concepto de talento académico o desempeño sobresaliente, pues estos últimos se expresan o emergen una vez el potencial ha contado con las oportunidades y las experiencias mínimas necesarias para ello.

\section{Familia y nivel socioeconómico: relación con el desarrollo y el aprendizaje}

Para que los estudiantes con AC puedan transformar su potencial intelectual en talento académico, requieren de un entorno rico en oportunidades de aprendizaje y un contexto facilitador (Arancibia, 2009; Freeman, 2000; Velasco \& Quiroga-Garza, 2018), donde cuenten con condiciones sociales, familiares y/o educativas que posibiliten la expresión o desarrollo del potencial en latencia (Covarrubias, 2018; Yun, 2020).

La familia y la sociedad afectan el desarrollo de las personas. En el caso de la sociedad, la interacción es influida por el tipo de barrio, la clase social, la etnia y las redes sociales con que cuenta la persona; mientras que en la familia, la interacción estaría caracterizada por la presencia o ausencia de estabilidad familiar, situación económica y recursos culturales (Martell, 2018).

\section{Familia}

Las personas con capacidades excepcionales tienen un potencial innato y latente que, para desarrollarse, requiere tanto de ambición y voluntad de desarrollarlo, como de entornos que brinden oportunidades para lograrlo (Wai, 2014), entre ellos la familia, la cual se constituye como un primer 
sistema donde comienza el desarrollo de la AC (Freeman, 2015; López-Aymes et al., 2013).

La familia es el primer espacio psicosocial donde ocurre el desarrollo humano (Elsner et al., 2002), en tanto institución, es clave en la socialización y desarrollo de las personas, cumpliendo tareas como el establecimiento de normas básicas para asegurar la convivencia en sociedad, la transmisión de valores, tradiciones y cultura, y la formación de la autonomía e identidad (Fajardo et al., 2017). Cada familia cumple el rol de transmitir las expectativas y las costumbres del entorno sociocultural en que se encuentra, al mismo tiempo que transmite sus expectativas y costumbres propias, donde sus integrantes interactúan entre sí, establecen modelos de comportamiento, objetivos y metas y se apoyan mutuamente (Freeman, 2015; López, 2003, citado en Ordaz \& Acle, 2012). En ese sentido, el rol que asume cada integrante estaría influido por la organización, la comunicación y el tipo de relaciones que se dan en ella (Velasco \& Quiroga-Garza, 2018).

Existe evidencia que muestra que los logros, las perspectivas y el desarrollo de los niños están influidos por el estilo de vida y riqueza cultural de sus familias (Fajardo et al., 2017; Freeman, 2000).

Bronfenbrenner (1985) afirma que los niños crecen en diversos contextos ecológicos que afectan su desarrollo, encontrándose entre algunos factores el tamaño de la familia, el nivel de escolarización, el estatus ocupacional de los padres, entre otros, existiendo familias donde los niños son nutridos y criados por sus padres en casa, mientras que, en otras, los niños son cuidados fuera de casa. Hay familias que viven en la pobreza y otras son pudientes económicamente. También están aquellas donde las madres, padres o cuidadores trabajan gran parte del día, mientras que en otras estos están en casa cuando los niños regresan de la escuela. Algunos niños crecen en vecindarios homogéneos étnicamente, mientras que otros no. Del mismo modo, hay niños que viven en circunstancias emocionalmente pobres en casa, donde existen problemas económicos, altos niveles de conflicto entre los padres o rotación de adultos cuidadores en sus vidas (Brofenbrenner, 1985; Freeman, 2015).

La clase social-ocupacional de los padres en la familia también sería un factor que impacta en el desarrollo y educación de los estudiantes, a medida que el nivel educativo y económico familiar es más alto, se encontrarían mejores resultados académicos, lo cual estaría dado por la relación entre el nivel cultural o educativo y el nivel ocupacional de los padres, y por las posibilidades materiales a las cuales las familias podrían acceder según su ingreso familiar (Fajardo et al., 2017). Además, el nivel de formación alcanzado por los padres conlleva a ser parte de un determinado nivel social que ofrece al niño distintos estímulos para el estudio y mayores expectativas futuras (Hoffman et al., 1995).

En Zapata-Zabala et al. (2012), se presentan diversos estudios que dan cuenta de que la baja escolaridad de los padres estaría relacionada con la baja calidad y escasez de estrategias que favorezcan el éxito escolar. Los niños de madres con altos niveles educativos cuentan con mayores niveles de conocimiento matemático y habilidades de lectura al ingresar al jardín de infantes, del mismo modo, como resultado de la exposición diferencial e informal de los padres al mundo natural, existen diferencias entre estudiantes de familias de niveles socioeconómicos bajos y altos respecto del conocimiento de las ciencias en el jardín infantil, brecha que persiste hasta la escuela primaria (Olszewski-Kubilius \& Corwith, 2018).

La posición social, la vida cultural y las oportunidades para aprender que ofrece el entorno a niños y niñas estarían relacionados con el nivel de formación alcanzado por los padres. Esto tendría un rol fundamental en la inteligencia y el rendimiento escolar dada la posición económica y cultural que conlleva pertenecer a cierto nivel social, los estímulos que dicho nivel ofrece y las expectativas depositadas en ellos. En la familia predominarán temas de 
conversación diferentes y códigos lingüísticos definidos dependiendo del nivel intelectual de los padres, pudiéndose encontrar que en familias de nivel de estudios medio-alto, se da un continuo de formación en el hogar que favorece un buen desarrollo académico en el estudiante, mientras que, en las familias donde los padres se han criado en circunstancias de pobreza cultural, estos no estarían familiarizados con una fácil comunicación verbal (Freeman, 2000; Mingorance \& Estebaranz, 1995).

\section{Nivel socioeconómico}

En los últimos años se ha producido un incremento en el estudio del efecto que tiene el nivel socioeconómico (NSE) en el desempeño cognitivo (Arán, 2012). Recientes investigaciones neurocientíficas dan cuenta de que los efectos de la pobreza llegan de manera temprana, afectando la salud y el desarrollo físico, cognitivo y afectivo de los niños, producto de la falta de acceso a servicios humanos de calidad y a la carencia de recursos (Plucker \& Peters, 2018), evidenciando que la clase social puede afectar negativa y significativamente el desarrollo temprano (Thomas, 2018).

La pobreza afecta diversos aspectos de la vida de los niños, entre ellos las oportunidades educativas, su seguridad básica y la transformación del potencial de talento académico en talento académico (OlszewskiKubilius \& Corwith, 2018), conformando estructuras sociales desiguales que afectan el desarrollo de habilidades cognitivas centrales para un buen desempeño (Cohen et al., 2008; Ramírez et al., 2011).

Diversos estudios dan cuenta de que estudiantes de familias del NSE bajo tienen un desempeño empobrecido en comparación a aquellos de familias del NSE más alto (Ramírez et al., 2011), encontrando diferencias en puntuaciones en tareas que valoran el nivel de inteligencia, el rendimiento académico, las habilidades lingüísticas, la atención, la memoria y las funciones ejecutivas entre niños de familias de ingresos medios y bajos, siendo inferiores los resultados en estos últimos (Arán, 2012; Emma et al., 2019).
Respecto de las habilidades lingüísticas, los efectos de la pobreza pueden verse desde edades muy tempranas, estimándose que, a la edad de cuatro años, los niños de familias de ingresos más altos están expuestos a 30 millones de palabras más que aquellos que provienen de familias de menores recursos. Los niños que provienen de familias con un NSE bajo, desarrollarían dichas habilidades de manera enlentecida, presentando mayor riesgo de desarrollar dificultades en el área lectora, muestran un desempeño inferior en tareas de lenguaje comprensivo y nivel de vocabulario en comparación a niños del NSE medio, afectando negativa y significativamente su desarrollo cognitivo (Arán, 2012; Espinoza, 2020; Olszewski-Kubilius \& Corwith, 2018).

Los déficits cognitivos y el impacto negativo en el rendimiento se podrían explicar, en parte, por las privaciones socioeconómicas de los hogares de los estudiantes en coexistencia con un acceso reducido a recursos que promuevan, entre algunos aspectos, la estimulación cognitiva y la salud (Hamilton et al., 2018; Zapata-Zabala et al., 2012). La nutrición también juega un rol importante. Niños de familias pobres ven afectada su capacidad de aprendizaje producto de una nutrición deficiente, haciéndose evidente que un niño que cuenta con una mejor alimentación tendrá un funcionamiento más eficaz a nivel biológico y, por ende, un mayor nivel de funcionamiento mental (Olszewski-Kubilius \& Corwith, 2018; Freeman, 2000).

Por otro lado, estudiantes que provienen de familias del NSE bajo están expuestos a una mayor cantidad de eventos estresantes (Arán, 2011). Un NSE bajo y la situación de pobreza significan un estado de vulnerabilidad para el desarrollo neurocognitivo (Sheridan et al., 2012) dadas las diversas circunstancias desfavorables, propias del entorno sociocultural, que el niño debe enfrentar. En este sentido, el desarrollo cerebral puede verse afectado de diversas maneras, entre ellas: el impacto negativo en el desarrollo de la corteza prefrontal, la cual está fuertemente asociada a las funciones ejecutivas (Dike, 
2017; Sheridan et al., 2012); las modificaciones en la activación de redes neuronales implicadas en la resolución de tareas que son propias de pruebas cognitivas, afectando negativamente su desempeño (Hermida et al., 2010); el hipocampo afectado negativamente por elevados niveles de cortisol producto de experiencias de estrés crónico y trauma, afectando procesos y funciones centrales para el aprendizaje como la atención y la memoria (Meléndez \& Solano, 2017); el eje hipotalámico-hipofisiarioadrenal, el cual tiene gran impacto en la salud física y socioafectiva en la adultez (Miller et al., 2011, citados en Gelabert, 2016); el lenguaje, vinculado al lóbulo temporal y frontal, y volumen disminuido de materia gris en el lóbulo frontal y parietal, afectando procesos de aprendizaje (Dike, 2017).

Ahora bien, las brechas entre niños de familias de bajos y altos ingresos en el rendimiento académico, no solo responden a la desigualdad en los ingresos, sino también a las diferencias entre familias en la inversión en oportunidades educativas para los niños. Las familias de los deciles más altos invierten entre cinco a siete veces más en sus hijos que aquellas de los deciles más pobres, incurriendo en gastos en guarderías de calidad, en educación formal temprana y actividades de enriquecimiento (Kornrich \& Furstenberg, 2013).

Según Santana (2010), las inequidades en acceso y calidad en educación formal serían un problema social y ético, que afectaría significativamente a los estudiantes con alto potencial provenientes de familias del NSE bajo. Si bien se ha comprobado que hay estudiantes con AC en todos los estratos socioeconómicos, son los dones de estudiantes de familias del NSE bajo los que son gravemente descuidados en el sistema educativo actual, pues provienen de familias que carecen de los recursos y conocimientos necesarios para aprovechar las oportunidades limitadas que existen para sus hijos. Estos estudiantes no cuentan con padres que puedan concentrarse en llevarlos a las aulas adecuadas con los mejores docentes, es probable que los niños se encuentren en escuelas con dificultades con docentes y administrativos que no identifican a los niños con AC o que no cuentan con los recursos para apoyarlos, por lo que los niños cuentan con menos oportunidades de aprender y desarrollar habilidades académicas en comparación a sus pares de ingresos más altos, principalmente en contextos externos a la escuela, y tienden a tener resultados más bajos en evaluaciones de rendimiento académico y capacidad cognitiva, siendo esto una barrera para su identificación y viéndose obligados a valerse por sí mismos (Finn, 2014; Hamilton et al., 2018; LópezAymes et al., 2014). En este sentido, Feinstein (2003) afirma que las diferencias en el NSE pueden limitar los logros educativos y cognitivos de niños con AC, siendo desfavorecidos aquellos que provienen de familias con NSE bajo (Thomas, 2018).

Sobre la calidad de los planes de estudios existirían brechas en la rigurosidad de los planes al que pueden acceder familias del NSE bajo y alto, siendo los estudiantes de familias del NSE elevado quienes reciben una instrucción y contenido más riguroso: en el nivel de experiencia y rotación de sus docentes, el tamaño de la clase, el acceso a tecnologías, la participación de los padres en eventos escolares, entre otros (Barton \& Coley, 2009).

Los estudiantes con AC provenientes de familias de clase media-alta y con buenas conexiones, suelen recibir una educación más sólida, pues el sistema educativo está diseñado para que cuenten con experiencias de aprendizaje que atiendan a sus necesidades educativas especiales, pudiendo encontrar espacios educativos de mejor calidad con docentes más capacitados (Finn, 2014) y los padres suelen invertir en oportunidades de estimulación y educación para sus hijos (López-Aymes et al., 2014).

Por otro lado, los ingresos familiares afectan el lugar y entorno donde las familias pueden vivir, su acceso a recursos comunitarios y educativos que apoyen el óptimo desarrollo infantil, el contacto con adultos que actúen como modelos y puedan ayudarles 
a visualizar un camino hacia la educación superior y carreras profesionales, y la seguridad en su barrio (Olszewski-Kubilius \& Corwith, 2018). En ese sentido, la elección de escuela y la calidad de esta se ve afectada por la pobreza, habiendo diversos estudios que dan cuenta de que la segregación escolar se ve fuertemente influida por la libre elección de escuelas, siendo los sectores más favorecidos los que tienen mayores beneficios (Córdoba et al., 2020; Olszewski-Kubilius \& Corwith, 2018).

\section{Segregación en educación}

En Latinoamérica, uno de los mayores desafíos y preocupaciones de la educación es resolver los problemas de inequidad de los sistemas educativos, siendo la segregación uno de los indicadores que la caracterizan (Morales, 2014; Murillo \& MartínezGarrido, 2017), habiendo en el sistema educativo chileno altos niveles de segregación (Carrasco et al., 2014).

La segregación escolar es un indicador de inequidad que refiere a una distribución desigual de los estudiantes en los establecimientos educativos en función de sus características sociales y/o personales, como el país de nacimiento, el género, y el NSE y cultural de las familias (Córdoba et al., 2020; Murillo \& Martínez, 2017; Murillo \& Martínez-Garrido, 2017). En este fenómeno confluyen diversas variables: están aquellas de índole contextual, relacionadas con el entorno regulatorio de los sistemas como son las tendencias demográficas; las de índole institucional, referidas a las normativas y regulaciones de los sistemas educativos y a la medida en que favorecen o dificultan la conformación de escuelas heterogéneas, como pueden ser las políticas institucionales; y las de índole sociocultural, relacionadas con las preferencias y apreciaciones de las familias al escoger una institución educativa (Bellei et al., 2018; Murillo et al., 2018).

Siguiendo los análisis realizados por diferentes estudiosos del tema (Canals et al., 2019; Gutiérrez \& Zamorano, 2018; Rodríguez et al., 2020), se puede sostener que en Chile hay escuelas donde se concentran estudiantes provenientes de familias de menores recursos socioeconómicos o de NSE bajo. Estas escuelas suelen ser financiadas por el estado y administradas por las municipalidades o por alguna corporación sin fines de lucro. Asimismo, hay escuelas que concentran familias de NSE medio, las cuales suelen ser de tipo particular subvencionado, financiadas por subvención estatal y aportes de las familias con financiamiento compartido. Por último, hay escuelas que concentran familias con mayores posibilidades socioeconómicas o NSE alto, las cuales suelen ser de tipo particular pagado o no subvencionado, financiadas completamente por las familias (Córdoba et al., 2020; Murillo \& Martínez, 2017; Sillard et al., 2018).

En el año 2008 entra en vigencia en Chile la Ley de Subvención Escolar Preferencial (SEP), la cual busca compensar la desigualdad e inequidad que afectan a estudiantes de sectores más vulnerables, entregando recursos económicos adicionales a los establecimientos que los acogen y estableciendo una estrategia de mejoramiento educativo. Con ello, se tienen dos objetivos centrales: modificar el subsidio a la demanda asignando mayor cantidad de recursos a estudiantes más vulnerables y definir compromisos con los actores educativos con el propósito de mejorar la calidad de la educación brindada, otorgando mayor igualdad de oportunidades a los estudiantes del país y relevando el principio de equidad en educación (Canals et al., 2019; Carrasco et al., 2015; Gutiérrez \& Zamorano, 2018; Raczynski et al., 2013; Valenzuela et al., 2013; Valenzuela et al., 2015).

Para que el establecimiento educativo pueda participar del programa y contar con los recursos que ofrece la ley SEP, debe firmar un Convenio de Igualdad de Oportunidades con el Ministerio de Educación. En este convenio se compromete a mejorar los resultados en pruebas estandarizadas, rendir cuentas por los recursos recibidos, en el caso de aquellos establecimientos con copago se comprometen a no realizar cobros a las familias de estudiantes prioritarios, no seleccionar estudiantes, y desarrollar un Plan de 
Mejoramiento Educativo (PME) (Carrasco et al., 2015; Valenzuela et al., 2013; Valenzuela, 2015).

Aun cuando no es el propósito de la ley SEP, diversos actores del sistema educativo chileno plantean como expectativa sobre esta el incremento de matrículas de estudiantes en establecimientos particulares subvencionados. Estos establecimientos al tener mayores incentivos para recibir a estudiantes más vulnerables, generan mayor movilidad de estos hacia establecimientos con resultados educativos más elevados (Valenzuela et al., 2013). Este cambio de composición social en los establecimientos educativos implicaría que los estudiantes más vulnerables estén expuestos a un mejor efecto par al encontrarse con compañeros con mayor capital socioeconómico y cultural, así como también, favorecería a su proceso de aprendizaje al ser parte de una comunidad educativa con mejores capacidades directivas y pedagógicas (Valenzuela et al., 2015).

La experiencia en Chile evidencia que el sistema de libre elección favorece a aquellas familias de ingresos medios y altos, mas no a aquellas de bajos ingresos. Esto debido a que los estudiantes provenientes de familias de NSE alto son reclutados por establecimientos particulares no subvencionados, mientras que los estudiantes de NSE medio con más recursos y habilidades se concentran en las escuelas privadas subvencionadas, donde las instituciones que reclutan y seleccionan a sus estudiantes concentran la mayor cantidad de alumnos que provienen de familias en mejor situación socioeconómica mejorando así los resultados de la escuela. Por otro lado, los estudiantes de familias con menos recursos se concentran en escuelas públicas de bajo desempeño (Elacqua \& Fábrega, 2004; Gayo et al., 2019), generándose un mercado educativo segmentado donde quienes tienen más recursos pueden acceder a más y mejor, y quienes tienen pocos recursos acceden a poco y peor (Olave \& Veléz, 2017).

En el proceso de elección de establecimiento por parte de las familias, aquellas de contextos pobres se ven influidas por la cercanía, buscan una experiencia escolar agradable para sus hijos gracias al apoyo docente, el buen trato y apertura de la escuela, sin considerar los resultados de la prueba SIMCE. Así, buscarían una escuela donde se mantenga un clima de disciplina que pueda ofrecer a sus hijos un espacio donde las desventajas asociadas a vivir en un barrio estigmatizado sean disminuidas y donde se asegure la adquisición de aprendizajes (Córdoba et al., 2020; Gayo et al., 2019).

Las familias de nivel socioeconómico medio valoran positivamente los niveles de exigencia elevados, descartan escuelas en barrios percibidos como peligrosos para evitar el contacto de sus hijos con estudiantes que representan la cultura marginal popular, consideran la composición social de las escuelas prefiriendo aquellas que seleccionan estudiantes y con copago, evalúan los impactos de la escuela a largo plazo pues aspiran a que sus hijos lleguen a la universidad y consideran los resultados de la prueba SIMCE en sus decisiones (Carrasco et al., 2015; Córdoba et al., 2020; Gayo et al., 2019).

En las familias de niveles socioeconómicos más acomodados, el resultado de la prueba SIMCE no es determinante, pues la elección de escuela está dada principalmente por la pertenencia a una comunidad con cierto estatus y cualidad socioeconómica; otorgan gran valor al desarrollo integral, al aprendizaje de una segunda lengua y a perpetuar privilegios por medio de la elección de establecimientos prestigiosos donde sus hijos compartan con otros niños cuyas familias tienen similar estilo de vida, redes de contacto y códigos culturales (Córdoba et al., 2020; Gayo et al., 2019).

A través de la ley SEP, los establecimientos educativos que atienden a estudiantes de familias de mayor vulnerabilidad socioeconómica reciben recursos adicionales para mejorar la calidad de la educación de quienes por esta situación de vulnerabilidad vean dificultado el proceso educativo (Asociación de Municipalidad de Chile [AMUCH], s/f). Esto incluye, según Raczynski et al. (2013), atención a la diversidad 
de estudiantes de manera especializada y adecuada a sus necesidades; lo que, según Valenzuela et al. (2015), generó el año 2011 una leve disminución en los niveles de segregación social en los establecimientos particulares subvencionados, concentrado principalmente entre los estudiantes de familias de NSE medio y medio-bajo. En este sentido, el trabajo realizado por Neilson (2013) da cuenta de que los estudiantes ubicados en el $40 \%$ más vulnerable entre los años 2007 y 2011, incrementaron sus puntajes en el sistema de medición de la calidad de la educación en lectura y matemáticas. Lo mencionado, según el mismo autor, se explicaría tanto por el incremento en el desempeño de los establecimientos como por la recomposición social de las escuelas, donde la ley SEP facilitó el incremento de alternativas de elección de establecimiento educativo a las familias de sectores más vulnerables al eliminar las barreras que implican el sistema de copago. No obstante, Murillo et al. (2018) sostienen que los establecimientos educativos ubicados en los distintos sectores socioeconómicos siguen brindando una educación diferenciada. Es así que se da el fenómeno de que en los establecimientos ubicados en los sectores de mayores ingresos, la calidad de la educación que se brinda suele ser mayor a aquella que se entrega en los establecimientos de sectores más desfavorecidos o vulnerables.

Al medir la calidad de la educación a través de la prueba SIMCE, la cual aporta información de los estándares de aprendizaje adquiridos por los estudiantes en los diferentes niveles de enseñanza, se encuentran diferencias en los resultados según el NSE de las familias de los estudiantes de cada institución educativa y según el tipo de dependencia de dicha institución (público, particular subvencionado o particular no subvencionado). Con esto se evidencia que estudiantes de familias del NSE más alto o pertenecientes a establecimientos particulares subvencionados y no subvencionados, tienen puntajes más altos que aquellos de familias de NSE bajo o que pertenecen a establecimientos públicos. De manera que, a menor ingreso y nivel educacional de los padres, menores resultados en el puntaje SIMCE
(Barahona et al., 2018; Espinoza, 2020; Espinoza \& Rosas, 2019; Gayo et al., 2019; Hamilton et al., 2018).

Lo anterior puede explicarse desde diversas perspectivas, entre ellas, las bajas expectativas de los docentes, un plan de estudio menos exigente y con menor énfasis en la programación escolar y el rendimiento, dificultad para contratar y retener docentes eficaces con experiencia previa y la exposición a diversos factores de riesgo que impactan y comprometen el funcionamiento psicológico y calidad de vida de los estudiantes (Gómez et al., 2014; Hamilton et al., 2018; Morales, 2014), entre otros.

La segregación escolar impactaría negativamente en el aprendizaje y bienestar de los estudiantes por la asimetría en la calidad de la oferta educativa. El empobrecimiento de la socialización y convivencia en diversidad, dificulta la integración social y perpetúa la exclusión, discriminación (Duk \& Murillo, 2019; Murillo et al., 2018), segregación periférica y concentración espacial de la riqueza (Méndez \& Gayo, 2019).

Considerando el papel que cumplen las variables familiares y socioeconómicas en el desarrollo, bienestar y aprendizaje de los estudiantes y en la transformación del potencial intelectual en desempeño sobresaliente, así como la segregación social existente en el sistema educativo chileno, se consideró relevante comparar, de manera exploratoria, el potencial de talento académico o $\mathrm{AC}$ en estudiantes de quinto año básico a cuarto año de enseñanza media de la provincia de Concepción, Chile, provenientes de familias de nivel socioeconómico bajo, medio y alto, según el tipo de establecimiento educativo en el que estudian. Considerando que no se encontraron resultados de investigación empírica en el contexto chileno que den cuenta de las diferencias en el potencial intelectual entre estudiantes que provienen de familias de diferentes NSE, se propuso explorar si existen diferencias en la proporción de estudiantes con alta capacidad en tres tipos de establecimientos educativos chilenos de 
la provincia de Concepción, Chile. Se espera, a la luz de lo expuesto en relación al fenómeno de segregación escolar en Chile, encontrar diferencias en la proporción de estudiantes con AC según el tipo de establecimiento en que los participantes cursan estudios, concentrándose una mayor proporción de estudiantes con AC en establecimientos particulares pagados, seguidos de establecimiento particulares subvencionados y una menor proporción de estudiantes con AC en establecimientos públicos.

\section{Método}

\section{Diseño}

Estudio cuantitativo y exploratorio, de una sola medición, donde se compara el potencial intelectual en escolares de la provincia de Concepción, medido por el test de matrices progresivas de Raven. Además, se realizan comparaciones por sexo, nivel educativo y tipo de establecimiento.

\section{Participantes}

La muestra estuvo constituida por 650 niños, niñas y jóvenes de la provincia de Concepción, Chile, específicamente de las comunas de Talcahuano, Concepción y Chiguayante.

Del total de la muestra, un 51.53\% eran mujeres, un $47.84 \%$ eran hombres y un .61\% no reporta sexo. En cuanto al nivel educativo, participaron estudiantes desde quinto año básico a cuarto año de enseñanza media, de los cuales un $46.61 \%$ cursaba estudios en enseñanza básica, es decir, cursaban estudios entre quinto y octavo básico, y un 53.38\% en enseñanza media, vale decir, entre primer y cuarto año de enseñanza media.

Las edades de los participantes están en el rango de 11 a 17 años, aproximadamente; las edades fueron aproximadas según el nivel educativo en que se encontraban los participantes al momento del estudio. Dada la distribución por nivel del sistema educativo chileno y la edad de ingreso al sistema escolar, lo esperable y normativo es que en quinto año básico los estudiantes tengan entre 11 y 12 años y en cuarto año de enseñanza media tengan entre 16 y 17 años.

Respecto del tipo de establecimiento, un 21.38\% cursaba estudios en un establecimiento público, vale decir, en un establecimiento que es financiado por el Ministerio de Educación de Chile. Por otro lado, un $40 \%$ cursaba estudios en un establecimiento particular subvencionado, es decir, en un establecimiento que recibe financiamiento tanto del Ministerio de Educación de Chile como de particulares o corporaciones. Por último, un $38.61 \%$ cursaba estudios en un establecimiento particular pagado, 0 sea, en un establecimiento que no recibe financiamiento estatal, sino que es financiado por particulares.

El nivel socioeconómico (NSE) de los participantes se clasificó en bajo, medio o alto, según el tipo de establecimiento en que el estudiante cursaba estudios dadas las preferencias y posibilidad de acceso de su familia. De manera que, cuando el estudiante cursaba estudios en un establecimiento público, se clasificó como estudiante proveniente de familia de NSE bajo. Si el participante cursaba estudios en un establecimiento particular subvencionado, fue clasificado como proveniente de familia de NSE medio. Finalmente, si cursaba estudios en un establecimiento particular pagado, se clasificó como proveniente de familia de NSE alto.

Se consideraron los siguientes criterios al momento de seleccionar la muestra. Por un lado, el incluir a estudiantes de los tres tipos de establecimiento educativo chileno según el tipo de financiamiento que reciben por parte del Estado, vale decir, se debe incluir estudiantes de establecimientos públicos, particulares subvencionados y particulares pagados. Asimismo, se deben incluir estudiantes hombres y mujeres. Por otro lado, se deben incluir estudiantes de todos los niveles educativos desde quinto año básico a cuarto año de enseñanza media. 


\section{Instrumento}

Test de matrices progresivas de Raven. Utilizado en este estudio para identificar estudiantes con y sin alta capacidad (AC), o potencial de talento académico. En este estudio se comprende la AC como la presencia de capacidades o aptitudes intelectuales que se expresan espontáneamente y sin entrenamiento previo, constituyéndose como un potencial intelectual superior al $90 \%$ de la población del mismo rango etario. En tanto potencial, requiere que la persona se comprometa con el aprendizaje, el entrenamiento y la práctica sistemática para transformar dicho potencial en desempeño sobresaliente o talento académico. Esta AC dota al estudiante de potencial para tener un desempeño sobresaliente en actividades académicas, el cual se expresará siempre y cuando sus necesidades educativas y socioafectivas sean atendidas, y esté en interacción con catalizadores ambientales que movilicen su motivación, esfuerzo y perseverancia (Navarro et al., 2021). Así, la AC se entiende como sinónimo de potencial de talento académico y se diferencia del concepto de talento académico o desempeño sobresaliente, pues estos últimos se expresan o emergen una vez el potencial ha contado con las oportunidades y experiencias mínimas necesarias para ello.

El test de matrices progresivas de Raven es un instrumento acultural, donde el participante debe completar 60 matrices lacunarias, distribuidas en 5 series de 12 ítems cada una, de complejidad creciente. El instrumento mide la habilidad intelectual general y busca identificar los sistemas del pensamiento del desarrollo intelectual (Raven \& Court, 1999). Es independiente al idioma, educación y capacidad verbal de quien la realiza, incluyendo personas analfabetas y sordomudas al tratarse de un instrumento no verbal (Alarcón et al., 2012; Cairo et al., 2000; Rossi-Casé et al., 2016).

En cuanto a sus características psicométricas, en un estudio realizado por Mera (2021), el test reporta un alfa de Cronbach de .947 puntos y un omega de McDonald's de .934 puntos para la escala total, dando cuenta de elevados niveles de confiabilidad como instrumento de medición. En un estudio previo, Fernández et al. (2004) reportan un alfa de Cronbach de .85 puntos.

Para clasificar a los participantes, se utilizan los baremos propuestos por Cayssials (1993), quien afirma que el test de matrices progresivas de Raven es un instrumento pertinente para hacer un diagnóstico de capacidad intelectual, proponiendo que a partir del percentil 90 se puede afirmar que la persona está dotada de una capacidad superior. Siguiendo a este auto, se clasificó como estudiante con AC, o potencial de talento académico, a aquellos que se ubicaron sobre el percentil 90; mientras que como estudiante sin AC, o potencial de talento académico, se clasificó a aquellos que puntuaron bajo el percentil 90, ubicando los puntajes brutos según edad en, sobre o bajo el percentil 90.

Si bien en un estudio realizado por Pérez (2018) se proponen nuevos baremos para población entre 13 y 64 años de edad, el equipo de investigación ha tomado la determinación de mantener los criterios propuestos por Cayssials, aun cuando estos no representan necesariamente la actual distribución del potencial intelectual. Esto, dado que los parámetros propuestos por Pérez (2018) no brindan información sobre criterios y diagnósticos para todo el rango etario que se incluye en la muestra de este estudio.

Es relevante destacar que el modelo teórico y conceptual a la base de este estudio, comprende la alta capacidad como un potencial. En tanto potencial, no implica necesariamente la expresión de talento académico ni un desempeño sobresaliente, sino que dota al estudiante de un potencial o recurso base para llegar a alcanzar dicho talento. Por tanto, en este estudio no es relevante el desempeño escolar de los participantes, es por ello que no son consideradas sus calificaciones ni su rendimiento académico. 


\section{Procedimiento}

Este estudio se enmarca en un proyecto de investigación liderado por la investigadora responsable de este estudio, el cual contempló la aplicación de cuatro instrumentos en total. Sin embargo, dado que para los propósitos de este estudio se consideran los datos recogidos por uno de ellos, que es el test de matrices progresivas de Raven, solo este es descrito en los instrumentos y considerado en los análisis.

Para acceder a la muestra, se contactó a directores y jefes técnicos de los establecimientos educativos para coordinar la autorización y aplicación de los instrumentos. Tanto directores como jefes técnicos consintieron la participación de sus estudiantes y estuvieron encargados de informar a las familias de los participantes. Se resguardaron los aspectos éticos atendiendo a la normativa vigente y como custodia de los datos quedó la investigadora principal de este estudio.

Se coordinó fecha y horario de aplicación de los instrumentos, la cual se llevó a cabo durante la jornada regular de clases en las aulas de los respectivos establecimientos educativos. Al iniciar la jornada, se informó a los estudiantes de las implicancias y la cualidad voluntaria de su participación, de manera que, quienes voluntariamente aceptaron participar, firmaron el documento de asentimiento informado y se mantuvieron en la sala de clases para iniciar el proceso de recolección de datos.

Finalizado el proceso de recolección y análisis de información, se realiza una devolución a los establecimientos educacionales, por medio de un seminario, así como se realiza la entrega de un boletín informativo, donde se da cuenta de los resultados y la información recogida por medio de los distintos instrumentos aplicados.

\section{Análisis de datos}

Los datos fueron analizados usando el programa estadístico SPSS en su versión 22. Se realizó un análisis de estadísticos descriptivos y un análisis de diferencias de medias de los puntajes de capacidad intelectual por sexo, entre estudiantes con y sin alta capacidad, por nivel educativo, y entre estudiantes de nivel socioeconómico bajo, medio y alto según el tipo de establecimiento en que cursan estudios, a través de ANOVA y $t$ de Student. 


\section{Resultados}

\section{Identificación de estudiantes con y sin alta capacidad o potencial de talento académico}

Se identifica como estudiante «con AC» a aquel cuyo puntaje en la escala de matrices progresivas de
Raven permite clasificarlo como un estudiante con potencial de talento académico; mientras que, como estudiante «sin AC», se clasifica para aquel cuyo puntaje lo sitúa como un estudiante sin potencial de talento académico (Tabla 1).

\section{Tabla 1}

Distribución de estudiantes con y sin alta capacidad (AC) en relación a la totalidad de la muestra, según tipo de establecimiento y nivel educativo

\begin{tabular}{|c|c|c|c|}
\hline \multicolumn{4}{|c|}{ Frecuencia y porcentaje de estudiantes con y sin alta capacidad } \\
\hline & & Con alta capacidad & Sin alta capacidad \\
\hline \multirow[t]{2}{*}{$N=650$} & $\mathrm{~F}$ & 97 & 553 \\
\hline & $\%$ & $14.2 \%$ & $85.07 \%$ \\
\hline \multicolumn{4}{|c|}{ Distribución de estudiantes con y sin alta capacidad según tipo de establecimiento } \\
\hline & & Con alta capacidad & Sin alta capacidad \\
\hline \multirow[t]{2}{*}{ Establecimiento público $(n=139)$} & F & 8 & 131 \\
\hline & $\%$ & $5.8 \%$ & $94.2 \%$ \\
\hline \multirow[t]{2}{*}{ Establecimiento particular subvencionado $(n=260)$} & $\mathrm{F}$ & 24 & 236 \\
\hline & $\%$ & $9.2 \%$ & $90.8 \%$ \\
\hline \multirow[t]{2}{*}{ Establecimiento particular pagado $(n=251)$} & $\mathrm{F}$ & 65 & 186 \\
\hline & $\%$ & $25.9 \%$ & $74.1 \%$ \\
\hline \multicolumn{4}{|c|}{ Distribución de estudiantes con y sin alta capacidad según nivel educativo } \\
\hline & & Con alta capacidad & Sin alta capacidad \\
\hline \multirow[t]{2}{*}{ Enseñanza básica $(n=303)$} & $\mathrm{F}$ & 43 & 260 \\
\hline & $\%$ & $14.2 \%$ & $85.8 \%$ \\
\hline \multirow[t]{2}{*}{ Enseñanza media $(n=347)$} & $\mathrm{F}$ & 54 & 293 \\
\hline & $\%$ & $15.6 \%$ & $84.4 \%$ \\
\hline
\end{tabular}

Medias y proporción de estudiantes con y sin alta capacidad o potencial de talento académico, según el tipo de establecimiento

Al comparar las medias capacidad intelectual según el tipo de establecimiento educativo, se identifican diferencias significativas entre las medias obtenidas del grupo particular pagado y las de los establecimientos particulares subvencionados y establecimientos públicos. La media más alta es en los establecimientos particulares pagados, seguidos de los establecimientos particulares subvencionados y la media más baja están en los establecimientos públicos. Para esto se realiza un ANOVA de una vía y el valor para el modelo es $F(2,647)=52.14 ; p<$ .001. Se realizó la prueba de Scheffe como análisis post hoc. Las diferencias se encuentran entre el 
grupo particular pagado $(M=48.81 ; D E=6.41 ; n=$ 251) y el particular subvencionado $(M=42.92 ; D E$ = 8.428; $n=260), p<.001$, IC95\% [4.24, 7.54] y entre el grupo particular pagado y el público $(M=$ 41.99; $D E=7.952 ; n=139), p<.001$, IC95\% [4.84, 8.79]. El tamaño del efecto calculado es $f=.37$, siendo un efecto mediano. Para ANOVA de una vía se considera efecto mediado entre .24 y .40 , y efecto grande sobre .40 puntos.

Por tanto, estudiantes de familias del NSE bajo, que cursan estudios en establecimientos públicos, son aquellos que puntúan más bajo en la escala de matrices progresivas de Raven. En orden ascendente, están aquellos de familias del NSE medio que cursan estudios en establecimientos particulares subvencionados, $\mathrm{y}$ quienes puntúan más alto son aquellos de familias del NSE alto, que cursan estudios en establecimientos particulares pagados o no subvencionados.

Lo anterior, también se observa en términos de proporción de estudiantes identificados con y sin AC o potencial de talento académico en los tres tipos de establecimientos (Tabla 2).

Tabla 2

Proporción de estudiantes identificados con y sin alta capacidad (AC), según el tipo de establecimiento en que cursan estudios

\begin{tabular}{cccc}
\hline $\begin{array}{c}\text { Dotación } \\
\text { intelectual }\end{array}$ & $\begin{array}{c}\text { Público } \\
(n=139)\end{array}$ & $\begin{array}{c}\text { Particular } \\
\text { subvencionado } \\
(n=260)\end{array}$ & $\begin{array}{c}\text { Particular } \\
\text { pagado } \\
(n=251)\end{array}$ \\
\hline Con alta capacidad & $5.8 \%$ & $9.2 \%$ & $\begin{array}{c}\text { Tendencia entre tres tipos de } \\
\text { establecimiento }\end{array}$ \\
Sin alta capacidad & $94.2 \%$ & $90.8 \%$ & $74.1 \%$ \\
\hline
\end{tabular}

La proporción de estudiantes identificados con AC es más baja en los establecimientos educativos públicos, donde cursan estudios estudiantes de familias del NSE bajo. De manera creciente, le siguen los establecimientos particulares subvencionados, donde estudiantes de familias del NSE medio cursan estudios. Y la mayor proporción de estudiantes con AC se encuentra en los establecimientos particulares pagados, donde estudiantes de familias de NSE alto cursan estudios.

\section{Media y proporción de estudiantes con y sin alta capacidad, o potencial de talento académico, de enseñanza básica y media en los distintos tipos de establecimiento}

Al comparar las medias capacidad intelectual según el tipo de establecimiento educativo y realizando el análisis por nivel de enseñanza, para el grupo de enseñanza básica la media más alta se encuentra en los establecimientos particulares pagados. De manera descendente, es seguido por los establecimientos públicos; $\mathrm{y}$, por último, los establecimientos particulares subvencionados. Para el nivel educativo medio, la media más alta también se encuentra en los establecimientos particulares pagados, seguido de manera descendente por los establecimientos particulares subvencionados y públicos. Para los análisis de ambos grupos (nivel de enseñanza medio y básico) se realiza un ANOVA de una vía.

El valor para el modelo del nivel educativo enseñanza básica es $F(2,300)=16.356 ; p<.001$. Se realizó la prueba de Scheffe como análisis post hoc. Las diferencias se encuentran entre el grupo particular pagado $(M=45.56 ; D E=6.328 ; n=117)$ y el particular subvencionado $(M=40.22 ; D E=8.70$; 
$n=117), p<.001$, IC95\% $[1.71,7.33]$ y entre el grupo particular pagado y el público $(M=41.04 ; D E$ $=7.232 ; n=69), p<.001$, IC95\% [2.92, 7.76]. El tamaño del efecto calculado es $f=.31$, siendo un efecto mediano.

El valor para el modelo del nivel educativo enseñanza media es $F(2,344)=47.477$; sig $<.001$. Se realizó la prueba de Scheffe como análisis post hoc. Las diferencias se encuentran entre el grupo particular pagado $(M=51.64 ; D S=5.003 ; n=134) \mathrm{y}$ el particular subvencionado $(M=45.12 ; D S=7.540 ; n$ = 143), sig < .001, IC95\% [4.48, 8.57] y entre el grupo particular pagado y el público $(M=42.93 ; D S=8.552$; $n=70), \operatorname{sig}<.001$, IC95\% [6.21, 11.22]. El tamaño del efecto calculado es $f=.46$, siendo un efecto grande.

$\mathrm{Al}$ analizar la proporción de estudiantes con AC de enseñanza básica y media en los distintos tipos de establecimientos educativos, se observa que, tanto a nivel básico como medio, la proporción de estudiantes con AC es mayor en establecimientos particulares pagados, seguidos de forma descendiente por los establecimientos particulares subvencionados y los establecimientos públicos.

Ahora bien, si se analizan las proporciones de estudiantes con AC en los distintos tipos de establecimiento según el nivel educativo de sus estudiantes, se encuentra que, tanto en los establecimientos públicos como en los particulares subvencionados, hay una mayor proporción de estudiantes de enseñanza básica con AC que estudiantes de enseñanza media con AC. Mientras que, en los establecimientos particulares pagados, hay una mayor proporción de estudiantes de enseñanza media con AC que de enseñanza básica con AC (Tabla 3).

Tabla 3

Proporción de estudiantes con y sin alta capacidad (AC), según nivel educativo en cada tipo de establecimiento

\begin{tabular}{|c|c|c|c|c|c|}
\hline \multirow{2}{*}{$\begin{array}{c}\text { Nivel } \\
\text { educativo }\end{array}$} & \multirow{2}{*}{ Situación } & \multicolumn{3}{|c|}{ Tipo de establecimiento } & \multirow{2}{*}{$\begin{array}{l}\text { Tendencia entre } \\
\text { tres tipos de } \\
\text { establecimiento }\end{array}$} \\
\hline & & $\begin{array}{l}\text { Público } \\
(n=139)\end{array}$ & $\begin{array}{c}\text { Particular } \\
\text { subvencionado } \\
(n=260)\end{array}$ & $\begin{array}{l}\text { Particular } \\
\text { pagado } \\
(n=251)\end{array}$ & \\
\hline \multirow{3}{*}{$\begin{array}{l}\text { Enseñanza básica } \\
\qquad(n=303)\end{array}$} & Con $\mathrm{AC}^{*}$ & $7.2 \%$ & $12 \%$ & $20.5 \%$ & \\
\hline & $\operatorname{Sin} A C$ & $92.8 \%$ & $88 \%$ & $79.5 \%$ & \\
\hline & Sub-total & $100 \%(n=69)$ & $100 \%(n=117)$ & $100 \%(n=117)$ & \\
\hline \multirow{3}{*}{$\begin{array}{l}\text { Enseñanza media } \\
\qquad(n=347)\end{array}$} & Con AC & $4.3 \%$ & $7 \%$ & $30.6 \%$ & \\
\hline & $\operatorname{Sin} A C$ & $95.7 \%$ & $93 \%$ & $69.4 \%$ & \\
\hline & Sub-total & $100 \%(n=70)$ & $100 \%(n=143)$ & $100 \%(n=134)$ & \\
\hline
\end{tabular}

Esto da cuenta, por un lado, que tanto a nivel básico como medio hay una mayor proporción de estudiantes con AC en establecimientos donde se asume un NSE alto y una menor proporción de estudiantes con AC en establecimientos donde se asume un NSE bajo. Por otro lado, se da cuenta que en establecimientos educativos donde se asume un NSE bajo o medio hay una mayor proporción de estudiantes con AC que cursan enseñanza básica, mientras que en establecimientos educativos donde se asume un NSE alto hay una mayor proporción de estudiantes con AC que cursan enseñanza media. 


\section{Discusión y conclusiones}

El propósito de este estudio es comparar de manera exploratoria, la distribución del potencial intelectual de estudiantes que cursan desde quinto año básico a cuarto año de enseñanza media en diferentes tipos de establecimiento educacional de la provincia de Concepción, Chile (público, particular subvencionado o particular pagado), asumiendo que el tipo de establecimiento en que cursan estudios es un predictor del nivel socioeconómico de la familia.

A la luz de los resultados obtenidos, es posible confirmar la presencia de diferencias estadísticamente significativas en el potencial de talento académico entre estudiantes de establecimientos públicos, particulares subvencionados y particulares pagados, y en estudiantes de enseñanza básica y enseñanza media que cursan estudio en los distintos tipos de establecimientos educativos.

En este sentido, al analizar las medias y la proporción de estudiantes con AC en los distintos tipos de establecimientos educativos, es posible observar indicios de segregación y distribución desigual del potencial de talento académico en los diferentes tipos de establecimiento educacional de los estudiantes participantes. Existe una mayor concentración de estudiantes con potencial de talento académico o AC en establecimientos educacionales en que participan estudiantes provenientes de familias del NSE alto y, por tanto, mayor recursos cultural e intelectual en el NSE alto y medio y una menor proporción de potencial de talento académico o AC en familias del NSE bajo.

Estos resultados podrían explicarse, por un lado, por lo propuesto por Elacqua y Fábrega (2004) y Gayo et al. (2019) respecto de la experiencia chilena con el sistema de libre elección. Por otro lado, podrían responder a los planteamientos de Ramírez et al. (2011), quienes exponen estudios que dan cuenta de que estudiantes del NSE bajo tienen un desempeño más bajo y empobrecido que aquellos del NSE más alto, pudiendo encontrar diferencias en puntuaciones de tareas relacionadas con la inteligencia (Arán,
2012; Emma et al., 2019). Esto podría estar dado por la experimentación de estrés crónico al que se ven expuestos los estudiantes provenientes de familias del NSE bajo (Evans \& English, 2002; Meléndez \& Solano, 2017).

Estos resultados dan cuenta de que, en los establecimientos educativos de los estudiantes participantes de este estudio, se ve reflejado y replicado un modelo que fomenta la segregación escolar y con ello favorece la inequidad e incremento en la brecha de oportunidades entre estudiantes provenientes de familias del NSE alto, medio y bajo. La mayor concentración de estudiantes con AC en establecimientos particulares pagados podría implicar mejores resultados en pruebas estandarizadas como la prueba SIMCE (Carrasco et al., 2015; Córdoba et al., 2020; Gayo et al., 2019).

Finalmente, la clase ocupacional y social de los padres en la familia es un factor de gran relevancia en la gestión de oportunidades educativas formales y no formales para nutrir el potencial intelectual y rendimiento de los estudiantes (Freeman, 2000; Mingorance \& Estebaranz, 1995; Olszewski-Kubilius \& Corwith, 2018; Zapata-Zabala et al., 2012). En este sentido, Fajardo et al. (2017) y Hoffman et al. (1995) señalan que a medida que el nivel educativo y económico familiar es más alto, se encontrarían mejores resultados académicos; lo cual estaría dado por la relación existente entre el nivel cultural o educativo y el nivel ocupacional de los padres, y por las posibilidades materiales a las cuales las familias podrían acceder según su ingreso familiar, de manera que la posición social, la vida cultural y las oportunidades para aprender, que el entorno ofrece a los niños, estarían relacionadas con el nivel de formación alcanzado por los padres.

Respecto del nivel educativo, es posible observar diferencias en la proporción de estudiantes con AC o potencial de talento académico de enseñanza básica y media, siendo mayor la proporción de estudiantes con $\mathrm{AC}$ en enseñanza media que en enseñanza básica. Al 
incorporar en el análisis el tipo de establecimiento, es posible observar que, tanto a nivel básico como medio, la mayor proporción de estudiantes con AC se concentra en establecimientos particulares pagados, seguidos de manera descendente por establecimientos particulares subvencionados $\mathrm{y}$, en último lugar, por establecimientos públicos. Más aún, es posible identificar que en establecimientos particulares pagados la proporción de estudiantes con AC es mayor en enseñanza media que en enseñanza básica; sin embargo, esto no ocurre en establecimientos particulares subvencionados y públicos, donde la proporción de estudiantes con AC es mayor en enseñanza básica que en enseñanza media. Estos resultados podrían dar cuenta de una movilidad de estudiantes con potencial de talento académico, quienes al pasar a la enseñanza media escogerían colegios particulares subvencionados o particulares pagados, los cuales según Barton y Coley (2009) tendrían un mejor plan curricular que los establecimientos públicos; existiendo, además, diferencias en el nivel de experiencia y rotación de sus docentes, en el tamaño de la clase, el acceso a tecnologías y participación de los padres en eventos escolares, entre otras variables, donde los más desfavorecidos serían los estudiantes de familias de NSE bajo.

Al ser la enseñanza media la etapa previa a la educación universitaria, pareciera que las familias de estudiantes con potencial de talento académico o AC que participaron de este estudio y que podían acceder a educación particular, ya sea pagada o subvencionada, escogieron este tipo de establecimiento para obtener una mejor preparación para el futuro. Esto guarda relación con lo propuesto por Finn (2014) respecto a que el sistema educativo está diseñado para que los estudiantes con AC provenientes de familias de clase media alta, reciban una educación más sólida, pudiendo encontrar espacios educativos de mejor calidad y con docentes más capacitados.

Por otra parte, la mayor proporción de estudiantes con potencial de talento académico que cursan enseñanza media y en colegios particulares pagados, podría explicarse siguiendo lo planteado por Arancibia (2009), Feinstein (2003), Finn (2014), Hamilton et al. (2018) y López-Aymes et al. (2014). Si bien el potencial de talento académico, o alta capacidad, se distribuye homogéneamente en la población y en todos los estratos socioeconómicos, podría ocurrir que al existir mayor capital cultural y un contexto extracurricular, social y familiar más enriquecido para los estudiantes de establecimientos particulares, o de familias de NSE alto, estos conservarían y fortalecerían su potencial en mayor medida que los estudiantes de escuelas públicas, provenientes de familias de NSE bajo, con un contexto extracurricular, social y familiar desventajado. Según Arancibia (2009), «lo más probable es que la mayor pérdida de talentos ocurra entre aquellos que tienen menos oportunidades, ya sea porque provienen de familias con carencias o porque asisten a escuelas que les entregan una educación de escasa calidad», lo cual también es planteado por Thomas (2018).

En conclusión, desde los resultados de este estudio, se puede sostener que el fenómeno de segregación se ve reflejado en los establecimientos educativos de los participantes, de manera que, en este grupo de estudiantes de quinto año básico a cuarto año de enseñanza media de la provincia de Concepción, aquellos con AC o potencial de talento académico se van concentrando en establecimientos particulares pagados, creándose contextos educativos y extracurriculares, sociales y familiares cada vez más enriquecedores para el grupo que puede acceder a estos establecimientos, a los que accederían solo unos pocos estudiantes con potencial de talento académico que provienen de establecimientos públicos, generalmente becados.

Entre las limitaciones de este estudio se encuentra su cualidad exploratoria. Dado que no se han incluido estudiantes provenientes de distintos tipos de establecimientos de otras regiones del país, no es posible generalizar estos resultados como una realidad a nivel nacional, por lo que se espera que en futuras líneas de investigación se considere abordar esta temática incluyendo la participación de un número 
mayor de participantes y que incluya distintas zonas y regiones del país.

Una implicancia práctica del estudio puede ser resaltar la importancia de generar espacios y estrategias educativas que permitan disminuir la brecha de oportunidades entre estudiantes con AC o potencial de talento académico que provienen de familias con altos y escasos recursos, y amortiguar los efectos de la segregación escolar; pudiendo ser los programas de enriquecimiento extracurricular complementarios a la educación regular, en los que participen estudiantes de colegios particulares pagados, particulares subvencionados y públicos, una alternativa importante para generar condiciones de equidad facilitando, además, la interacción y enriquecimiento extracurricular, social y familiar entre estudiantes, al menos para este grupo de personas.

\section{Conflicto de intereses}

Los autores informan que no existe ningún tipo de conflictos de intereses como morales, económicos, laborales y de investigación.

\section{Responsabilidad ética}

Los autores indican que para esta investigación no se han realizado experimentos en seres humanos ni en animales.

\section{Contribución de autoría}

Las autoras participaron en todo el desarrollo del artículo.

\section{Referencias}

Alarcón, C., Díaz, V., Hernández, J., \& Estrada, C. (2012). Estudio sobre la pertinencia del uso de las normas disponibles del Raven en adultos mayores chilenos. Psico-USF, 17(3), 387-395. https://doi.org/10.1590/ S1413-82712012000300005

Arán, V. (2011). Funciones ejecutivas en niños escolarizados: efectos de la edad y del estrato socioeconómico. Avances en Psicología Latinoamericana/Bogotá, 29(1),
98-113. https://revistas.urosario.edu.co/index.php/apl/ article/view/499/1477

Arán, V. (2012). Estrato socioeconómico y habilidades cognitivas en niños escolarizados: variables predictoras y mediadoras. PSYKHE, 21(1), 3-20. https://doi.org/ 10.4067/S0718-22282012000100001

Arancibia, V. (2009). La educación para alumnos con talentos: una deuda y una oportunidad para Chile. Dirección de Asuntos Públicos de la Pontificia Universidad Católica de Chile.

Asociación de Municipalidad de Chile. (s/f). Ley de Subvención Escolar Preferencial $N^{\circ} 20.248$ [presentación de diapositivas]. Cámara de diputadas y diputados de Chile. https://www.camara.cl/verDoc.as px?prmID=23075\&prmTIPO=DOCUMENTOCOMISION

Barahona, P., Veres, E., \& Barahona, M. (2018). Factores asociados a la calidad de la educación en Chile. Revista Internacional de Investigación en Ciencias Sociales, 14(1), 17-30. http://revistacientifica.uaa.edu.py/index. php/riics/article/view/482/pdf

Barton, P. E., \& Coley, R. J. (2009). Parsing the Achievement Gap II. Policy Information Report. Educational Testing Service. https://files.eric.ed.gov/fulltext/ED505163.pdf

Bellei, C., Contreras, M., Canales, M., \& Orellana, V. (2018). The Production of Socio-Economic Segregation in Chilean Education. School Choice, Social Class and Market Dynamics. En X. Bonal \& C. Bellei (Eds.), Understanding School Segregation. Patterns, Causes and Consequences of Spatial Inequalities in Education (pp. 221-240). Bloomsbury Academic.

Bronfenbrenner, U. (1985). Contextos de crianza del niño. Problemas y prospectiva. Infancia y aprendizaje, 8(29), 45-55. https://doi.org/10.1080/02103702.1985.10822058

Cairo, E., Cairo, E., Bouza, C., \& Solozabal, P. (2000). Algunas características y posibilidades del test de matrices progresivas de Raven. Revista Cubana de Psicología, 17(2), 95-105. http://pepsic.bvsalud.org/ $\mathrm{pdf} / \mathrm{rcp} / \mathrm{v} 17 \mathrm{n} 2 / 02 . \mathrm{pdf}$

Canals, C., Aguirre, C., Blanco, C., Fábrega, F., Mena, C., \& Paulus, N. (2019). El «voucher» a la chilena. Reflexiones sobre elección escolar y financiamiento educacional. Estudios Pedagógicos, 45(1), 137-150. https://doi.org/10.4067/S0718-07052019000100137 
Carrasco, A., Contreras, D., Elacqua, G., Flores, C., Mizala, A., Santos, H., Torche, F., \& Valenzuela, J. P. (2014). Informe de políticas públicas $N^{\circ}$ 3. Hacia un sistema escolar más inclusivo: Cómo reducir la segregación escolar en Chile. Espacio Público. https:// espaciopublico.cl/wp-content/uploads/2021/05/25.pdf

Carrasco, R., Pérez, M., \& Núñez, D. (2015). Hacia una distribución más equitativa de las oportunidades educativas: ¿cuál es el impacto de la política de Subvención Preferencial en el desempeño académico de los alumnos más vulnerables en Chile? Pensamiento Educativo. Revista de Investigación Educacional Latinoamericana, 52(1), 65-83.

Cohen, S., Cotini, N. E., Coronel, P., \& Caballero, V. (2008). Habilidades cognitivas en niños de contextos de pobreza. Psicodebate, 8, 17-36. https://doi.org/10.186 82/pd.v8i0.414

Córdoba, C., Laborda, A., \& Reyes, C. (2020). Preferencias de elección de escuela en dos casos de alta segregación escolar. REICE. Revista Iberoamericana sobre Calidad, Eficacia y Cambio en Educación, 18(4), 325-344. https:/ /doi.org/10.15366/reice2020.18.4.013

Cornejo, C., \& Benavides, E. (2013). Talento académico. Una aproximación al estado del arte. En C. Cornejo, P. Morales, E. Saavedra, \& G. Salas (Eds.), Aproximaciones en psicología educacional: diversidades ante la contingencia actual (pp. 54-67). Universidad Católica del Maule.

Covarrubias, P. (2018). Del concepto de aptitudes sobresalientes al de altas capacidades y el talento. IE Revista de investigación educativa de la REDIECH, 9(17), 53-67. https://doi.org/10.33010/ie_rie_rediech.v 9i17.123

Dike, V. E. (2017). Poverty and Brain Development in Children: Implications for Learning. Asian Journal of Education and Training, 3(1), 64-68. https://files.er ic.ed.gov/fulltext/EJ1147369.pdf

Duk, C., \& Murillo, F. J. (2019). Segregación escolar y meritocracia. Revista Latinoamericana de Educación Inclusiva, 13(1), 11-13. https://doi.org/10.4067/S071873782019000100011

Elacqua, G., \& Fábrega, R. (2004). El consumidor de la educación: El actor olvidado de la libre elección de escuelas en Chile. Editorial PREAL. http://www.opech.
cl/bibliografico/Doc_Financiamiento/consumidor_ educacion.pdf

Elsner, P., Montero, M., Reyes, C., \& Zegers, B. (2002). La familia: Una aventura. Editorial Alfaomega.

Emma, E., Arán-Filippetti, V., \& Vargas-Rubilar, J. (2019). Estrato socioeconómico y funcionamiento ejecutivo: su relación con las competencias académicas en edad escolar. Panamerican Journal of Neuropsychology, 13(3), 80-93.

Espinoza, M. V. (2020). Precursores del lenguaje escrito en niños de distintos niveles socioeconómicos en Chile [Tesis de doctorado, Pontificia Universidad Católica de Chile]. Repositorio UC. https:// repositorio.uc.cl/handle/11534/50028

Espinoza, V., \& Rosas, R. (2019). Diferencias iniciales en el proceso de acceso al lenguaje escrito según nivel socioeconómico. Perspectiva Educacional, 58(3), 2345. https://doi.org/10.4151/07189729-vol.58-iss.3-art.955

Evans, G. W., \& English, K. (2002). The Environment of Poverty: Multiple Stressor Exposure, Psychophysiological Stress, and Socioemotional Adjustment. Child Development, 73(4), 1238-1248. https://doi.org/10.1111/1467-8624.00469

Fajardo, F., Maestre, M., Felipe, E., León, B., \& Polo, M. I. (2017). Análisis del rendimiento académico de los alumnos de educación secundaria obligatoria según las variables familiares. Educación XX1, 20(1), 209-232. https://doi.org/10.5944/educxx1.17509

Feinstein, L. (2003). Inequality in the Early Cognitive Development of British Children in the 1970 Cohort. Económica, 70(277), 73-97. https://doi.org/10.1111/ 1468-0335.t01-1-00272

Fernández, M., Ongarato, P., Saavedra, E., \& Casullo, M. (2004). Test de Matrices Progresivas, Escala General: Un análisis psicométrico. Evaluar, 4(1), 50-69. https:// doi.org/10.35670/1667-4545.v4.n1.598

Finn, C. E. (2014). Gifted, Talented, and Underserved. National Affairs, 18, 50-62. https://www.nationalaffairs. com/publications/detail/gifted-talented-andunderserved

Freeman, J. (2015). Por qué algunos niños con altas capacidades son notablemente más exitosos en la vida que otros con iguales oportunidades y habilidad. 
Revista de Educación, 368, 255-278. https://doi.org/ 10.4438/1988-592X-RE-2015-368-291

Freeman, J. (2000). Families, the essential context for gifts and talents. En K. A. Heller, F. J. Monks, R. Sternberg., \& R. Subotnik (Eds.), International handbook of research and development of giftedness and talent (pp. 573-585). Pergamon Press.

Gagné, F. (2015). De los genes al talento: la perspectiva DMGT/CMTD. Revista de Educación, 368, 12-39. https://doi.org/10.4438/1988-592X-RE-2015-368-289

Gayo, M., Otero, G., \& Méndez, M. L. (2019). Elección escolar y selección de familias: reproducción de la clase media alta en Santiago de Chile. Revista Internacional de Sociología, 77(1), e120. https://doi.org/10.3989/ ris.2019.77.1.17.310

Gelabert, M. (2016). Contextos de pobreza y desatención en la infancia: determinantes en la desregulación emocional y la conducta no adaptativa. Revista d'Afers Socials, 7, 49-65.

Gómez, V., Muñoz, C., Silva, I., González, M. P., Guerra, P., \& Valenzuela, J. (2014). Creencias y oportunidades de aprendizaje en la práctica educativa en contextos de pobreza. Perfiles Educativos, 36(144), 173-188.

Gómez-Arizaga, M. P., Trufello, A., \& Kraus, B. (2019). Percepciones parentales respecto a la experiencia académica y social de sus hijos con altas capacidades intelectuales. Perspectiva Educacional, 58(3), 156-177. https://doi.org/10.4151/07189729-vol.58-iss.3-art.837

González, M. T., \& Domingues, S. (2015). ¿Existen indicadores para identificar el talento? Aula, 21, 21-32. https://doi.org/10.14201/aula2015212132

Gutiérrez, E., \& Zamorano, R. (2018). Formas de inclusiónexclusión en el sistema educativo chileno: el movimiento estudiantil secundario 2006-2011. Revista Brasileira de Educação, 23, e230077. https://doi.org/ 10.1590/S1413-24782018230077

Hamilton, R., McCoach, D. B., Tutwiler, M. S., Siegle, D., Gubbins. E. J., Callahan, C. M., Brodersen, A. V., \& Mun, R. U. (2018). Disentangling the Roles of Institutional and Individual Poverty in the Identification of Gifted Students. Gifted Child Quarterly, 62(1), 6-24. https:// doi.org/10.1177/0016986217738053
Hermida, M. J., Segretin, M. S., Lipina, S. J., Benarós, S., \& Colombo, J. A. (2010). Abordajes neurocognitivos en el estudio de la pobreza infantil: consideraciones conceptuales y metodológicas. International Journal of Psychology and Psychological Therapy, 10(2), 205225. https://www.ijpsy.com/volumen10/num2/258/ abordajes-neurocognitivos-en-el-estudio-ES.pdf

Hoffman, L., Paris, S., \& Hall, E. (1995). Psicología del desarrollo hoy. McGraw Hill.

Kornrich, S., \& Furstenberg, F. (2013). Investing in Children: Changes in Parental Spending on Children, 1972-2007. Demography, 50(1), 1-23. https://doi.org/ 10.1007/s13524-012-0146-4

López-Aymes, G., Roger, S., \& Durán, G. (2014). Families of Gifted Children and Counseling Program: A Descriptive Study in Morelos, Mexico. Journal of Curriculum and Teaching, 3(1), 54-62. https://doi.org/10.5430/jct.v3n1p54

López-Aymes, G., Roger, S., \& Mercado, A. E. (2013). Niños y niñas con altas capacidades intelectuales y sus familias. En A. Bazán \& C. Butto (Coords.), Psicología y contexto educativos (pp. 53-116). Universidad Pedagógica Nacional.

Martell, G. (2018). Nivel socio - cultural de los padres de familia relacionados al nivel de desarrollo de las habilidades cognitivas en niños y niñas de 5 años de edad en la Institución Educativa Inicial $N^{\circ} 221$ distrito de Chazuta - 2016 [Tesis de maestría, Universidad Nacional de San Martín]. Repositorio Institucional Digital. http://hdl.handle.net/11458/3408

Meléndez, L., \& Solano, V. (2017). La desnutrición y el estrés van a la escuela: pobreza infantil y neurodesarrollo en América Latina. Innovaciones Educativas, 19(27), 5570. https://doi.org/10.22458/ie.v19i27.1955

Méndez, M. L., \& Gayo, M. (2019). Upper Middle Class Social Reproduction: Wealth, Schooling, and Residential Choice in Chile. Springer.

Mera, S. M. (2021). Propiedades psicométricas del test de matrices progresivas estándar de Raven (SPM) en estudiantes universitarios [Tesis de pregrado, Universidad Cooperativa de Colombia]. Repositorio Institucional UCC. http://hdl.handle.net/20.500.12494/ 34781 
Mingorance, P., \& Estebaranz, A. (1995). Factores socioculturales que influyen en la educación: la aportación del modelo etnográfico. Bordón. Revista de pedagogía, 47(4), 417-426.

Miguel, A., \& Moya, A. (2011). Conceptos generales del alumno de altas capacidades. En J. C. Torrego (Coord.), Alumnos con altas capacidades y aprendizaje cooperativo. Un modelo de respuesta educativa (pp. 13-33). Fundación Pryconsa \& Fundación SM. http:// www3.uah.es/convivenciayaprendizajecooperativo/wpcontent/uploads/2016/05/Alumnos-con-altascapacidades-y-aprendizaje-cooperativo-LibroTorrego.pdf

Morales, M. (2014). Estudiantes de sectores vulnerables con alto rendimiento escolar y su desempeño en variables intelectuales, resiliencia, autoestima y bienestar psicológico. Revista de Investigación en Psicología, 17(1), 93-105. https://doi.org/10.15381/ rinvp.v17i1.8973

Murillo, F. J., Duk, C., \& Martínez-Garrido, C. (2018). Evolución de la segregación socioeconómica de las escuelas de América Latina. Estudios Pedagógicos, 44(1), 157-179. https://doi.org/10.4067/S071807052018000100157

Murillo, J., \& Martínez, C. (2017). Segregación social en las escuelas públicas y privadas en América Latina. Educación y Sociedad, 38(140), 727-750. https:// doi.org/10.1590/ES0101-73302017167714

Murillo, F. J., \& Martínez-Garrido, C. (2017). Estimación de la magnitud de la segregación escolar en América Latina. Revista Internacional de Investigación en Educación, 9(19), 11-30. https://doi.org/10.11144/ Javeriana.m9-19.emse

Navarro, G., Flores-Oyarzo, G., \& Rivera, J. (2021). Relación entre autoestima y regulación emocional en estudiantes con alta capacidad que participan de un programa de enriquecimiento extracurricular chileno. Calidad en la Educación, 55, 6-40. https://doi.org/ 10.31619/caledu.n55.1007

Neilson, C. (2013). Targeted Vouchers, Competition Among Schools, and the Academic Achievement of Poor Students [documento de trabajo]. Yale University. https://economics.sas.upenn.edu/sites/default/files/ filevault/event_papers/Neilson_2013_JMP_current.pdf
Olave, J. M., \& Veléz, C. (2017). Las familias entre fines educativos y económicos. El caso de Chile. Alteridad. Revista de Educación, 12(1), 43-54. http://doi.org/ 10.17163/alt.v12n1.2017.04

Olszewski-Kubilius, P., \& Corwith, S. (2018). Poverty, Academic Achievement, and Giftedness: A Literature Review. Gifted Child Quarterly, 62(1), 37-55. https:// doi.org/10.1177/0016986217738015

Ordaz, G., \& Acle, G. (2012). Perfil psicosocial de adolescentes con aptitudes sobresalientes de un bachillerato público. Electronic Journal of Research in Educational Psychology, 10(28), 1267-1298. https:/ /doi.org/10.25115/ejrep.v10i28.1534

Pérez, J., Borges, Á., \& Rodríguez, E. (2017). Conocimientos y mitos sobre altas capacidades. Talincrea: Revista talento, inteligencia y creatividad, 4(1), 40-51. https:/ /www.cucs.udg.mx/talineng/sites/default/files/ adjuntos/03_06/06_Conocimientos.pdf

Plucker, J. A., \& Peters, S. J. (2018). Closing Poverty-Based Excellence Gaps: Conceptual, Measurement, and Educational Issues. Gifted Child Quarterly, 62(1), 5667. https://doi.org/10.1177/0016986217738566

Raczynski, D., Muñoz, G., Weinstein, J., \& Pascual, J. (2013). Subvención Escolar Preferencial (SEP) en Chile: un intento por equilibrar la macro y micro política escolar. REICE. Revista Iberoamericana sobre Calidad, Eficacia y Cambio en Educación, 11(2), 164193. https://revistas.uam.es/reice/article/view/2902

Ramírez, M., Devia, R. E., \& León, R. A. (2011). Pobreza y rendimiento escolar: estudio de caso de jóvenes de alto rendimiento. EDUCERE, 15(52), 663-672. http:// erevistas.saber.ula.ve/index.php/educere/article/view/ $13363 / 21921924453$

Raven, J. C., \& Court, J. H. (1999). Test de matrices progresivas. Manual. Paidós.

Renzulli, J. (2012). Reexamining the Role of Gifted Education and Talent Development for the $21^{\text {st }}$ Century: A FourPart Theoretical Approach. Gifted Child Quarterly, 56(3), 150-159. https://doi.org/10.1177/0016986212444901

Rodríguez, C., Espinosa, D., \& Padilla, G. (2020). Dónde quiero que estudien mis hijos/as: caracterización de la oferta educativa y sus niveles de demanda en Chile. Revista de Estudios y Experiencias en Educación, 
19(41), 57-70. https://doi.org/10.21703/rexe.20201941 rodriguez4

Rossi-Casé, L., Neer, R., Lopetegui, S., Doná, S., Biganzoli, B., \& Garzaniti, R. (2016). Test de Raven: actualización de baremos en adolescentes argentinos y análisis del efecto Flynn. Revista Iberoamericana de Diagnóstico y Evaluación, 42(2), 3-13. https://doi.org/10.21865/ RIDEP42_3

Santana, A. (2010). Relación familia escuela en contextos de pobreza. Posibilidades y limitaciones en los procesos educativos. Revista CUHSO, 20(2), 81-93. https://doi.org/10.7770/CUHSO-V20N1-ART322

Sheridan, M. A., Sarsour, K., Jutte, D., D’Esposito, M., \& Boyce, W. T. (2012). The Impact of Social Disparity on Prefrontal Function in Childhood. PloS ONE, 7(4), e35744. https://doi.org/10.1371/journal.pone.0035744

Sillard, M., Garay, M., \& Troncoso, I. (2018). Análisis al nuevo sistema de admisión escolar en Chile: la región de Magallanes como experiencia piloto. Calidad en la Educación, 49, 112-136. https://doi.org/10.31619/ caledu.n49.578

Thomas, M. S. (2018). A Neurocomputational Model of Developmental Trajectories of Gifted Children Under a Polygenic Model: When are Gifted Children Held Back by Poor Environments? Intelligence, 69, 200-212. https://doi.org/10.1016/j.intell.2018.06.008

Tourón, J. (2004). De la superdotación al talento: evolución de un paradigma. En C. Jiménez (Coord.), Pedagogía diferencial. Diversidad y equidad (pp. 369-400). Pearson Educación.

Valenzuela, J. P. (2015). El efecto de la SEP en la reducción de la segregación socioeconómica del sistema escolar chileno. Primeros resultados. Estudios de Política Educativa, 2. https://centroestudios.mineduc.cl/wp-
content/uploads/sites/100/2017/07/F811333-InformeFinal-Valenzuela-UCHILE.pdf

Valenzuela, J. P., Gómez, G., \& Allende, C. (2015, junio). «La Santa SEP», El efecto de la Ley de subvención escolar preferencial en la reducción de la segregación socioeconómica del sistema escolar chileno desde la mirada de los actores. V Congreso Nacional e Internacional de Estudios Comparados en Educación, Buenos Aires.

Valenzuela, J. P., Villarroel, G., \& Villalobos, C. (2013). Ley de Subvención Escolar Preferencial (SEP): algunos resultados preliminares de su implementación. Pensamiento Educativo: Revista de Investigación Educacional Latinoamericana, 50(2), 113-131.

Velasco, M. F., \& Quiroga-Garza, A. (2018). Factores sistémicos asociados a la experiencia escolar de adolescentes con alto potencial intelectual. Revista Mexicana de Investigación Educativa, 23(79), 10511074. https://www.comie.org.mx/revista/v2018/rmie/ index.php/nrmie/article/view/1188/1170

Wai, J. (2014). Experts are Born, then Made: Combining Prospective and Retrospective Longitudinal Data Shows that Cognitive Ability Matters. Intelligence, 45, 74-80. https://doi.org/10.1016/j.intell.2013.08.009

Yun, D. (2020). Rethinking Human Potential from a Talent Development Perspective. Journal of Education of the Gifted, 43(1), 19-37. https://doi.org/10.1177/016235321 9897850

Zapata-Zabala, M. E., Álvarez-Uribe, M. C., AguirreAcevedo, D. C., \& Cadavid-Castro, M. A. (2012). Coeficiente intelectual y factores asociados en niños escolarizados en la ciudad de Medellín, Colombia. Revista de Salud Pública, 14(4), 543-557. https:// revistas.unal.edu.co/index.php/revsaludpublica/article/ view/20522/40320 


\section{Gracia Navarro-Saldaña}

Universidad de Concepción, Chile

Doctora en Educación, magíster en Responsabilidad Social Corporativa, psicóloga, académica de la Universidad de Concepción y directora del Programa Talentos UdeC.

ORCID: https://orcid.org/0000-0002-2581-2510

Autora corresponsal: gnavarro@udec.cl

Gabriela Flores-Oyarzo

Universidad de Concepción, Chile

Psicóloga, coordinadora académica de inclusión del Programa Talentos UdeC.

ORCID: https://orcid.org/0000-0002-9061-8183

gabflores@udec.cl

María-Gracia González Navarro

Universidad de Concepción, Chile

Magíster en Psicología Educacional, psicóloga, colaboradora de investigación y asesora para el desarrollo docente del Programa Talentos UdeC.

ORCID: https://orcid.org/0000-0002-1499-5518

mgonzalez@udec.cl 